\title{
Renutrition après ingestion de régimes comprenant $10 \%$ de protéines de soja associées à diverses concentrations d'alginate ou de carraghénate de sodium. Effets sur la croissance et les paramètres lipidiques chez le rat *
}

\author{
J Mouécoucou, C Villaume **, HM Bau, JP Nicolas, L Méjean
}

\begin{abstract}
INSERM U 308, Unité de recherches sur les mécanismes de régulation du comportement alimentaire, 38, rue Lionnois, 54000 Nancy, France
\end{abstract}

(Reçu le 3 avril 1990; accepté le 22 mars 1991)

\begin{abstract}
Résumé - Des rats mâles de souche Wistar ont été nourris au cours de 3 périodes expérimentales consécutives. Au cours de la première période de 4 semaines, des régimes à $10 \%$ de protéines de caséine ou de farine déshuilée de soja additionnée ou non de $0,5,1,2$ ou $3 \%$ d'alginate ou de carraghénate de sodium et chauftée ont été utilisés. Au cours de la seconde période de 16 semaines, un régime standard à $17 \%$ de protéines a été fourni. Enfin, au cours de la dernière période, les mêmes régimes qu'en première période ont été dispensés, la seule différence résidait dans le taux de protéines porté à $20 \%$. Les poids corporels ont été mesurés durant toute l'étude à l'issue de laquelle les paramètres lipidiques sanguins ont été déterminés à jeun. Durant toute l'expérimentation, la présence d'alginate dans les régimes a été sans conséquence sur la croissance des rats, alors que l'ingestion des carraghénates a entraîné un retard de croissance à la fin de la première période expérimentale, retard non rattrapé après passage au régime standard. L'alginate a été sans effet sur la triglycéridémie sauf à la dose de $1 \%$ alors que le carraghénate s'est avéré hypotriglycéridémiant. Ni l'alginate ni le carraghénate n'ont modifié la cholestérolémie. L'ingestion de caséine par rapport à celle de farine de soja n'a pas provoqué l'augmentation du cholestérol sanguin généralement décrite. L'effet des carraghénates sur la croissance et la triglycéridémie pourrait être la conséquence de leurs propriétés physico-chimiques.
\end{abstract}

alginate / carraghénate de sodium / rat / triglycéridémie / croissance / soja

Summary-Refeeding after ingestlon of diets composed of $10 \%$ soyabean protein and varlous concentrations of alginates or carrageenans. Effects on growth and blood lipids of rats. Male 21-d-old Wistar rats were fed over 3 experimental periods. During the first period of 4 wk, diets contained $10 \%$ casein or defatted soy flour proteins, with or without $0.5,1,2$ or $3 \%$ sodium alginate or sodium carrageenan, and were heated. During the second period, they were fed a standard diet for 16 wk with $17 \%$ proteins, and during the third period, they received the same diet as in the first

\footnotetext{
* Présenté sous forme de poster au 3rd International Workshop on plant polysaccharides, Le Croisic, France (19-21/9/1990).

** Correspondance et tirés à part
} 
period, but with $20 \%$ proteins. Rat body weights were measured throughout the study period; plasma lipid levels were then determined after fasting. Presence of sodium alginate in the diet had no effect on growth, but rats fed carageenan presented growth retardation at the end of the experimental period, which was not altered by refeeding the standard diet. Sodium alginate did not modify rat triclyceridemia, except at the 1\% level. Carageenan had a hypotriglyceridemic effect. Alginate and carrageenan had no effect on blood cholesterol. Compared to soybean protein, casein intake did not increase plasma cholesterol levels as generally described. The effect of carragenans on growth and plasma triglyceride levels could be a result of their physico-chemical properties.

\section{alginate / sodium carraghenate / rat / plasma triglyceride level / growth / soya}

\section{INTRODUCTION}

La consommation sans cesse croissante de produits allégés en lipides et de viandes "végétales" nécessite l'utilisation d'agents de texture dans les procédés de fabrication. Leurs interactions avec les protéines permettent d'obtenir des produits d'une texture améliorée. Parmi ces agents texturants, sont employés des hydrocolloïdes alimentaires d'origine végétale tels que les alginates et les carraghénates de sodium (E401 et E407), issus d'algues marines.

La contribution grandissante des protéines végétales telles que celles du soja dans l'alimentation humaine est due non seulement au faible coût de ces protéines, mais également à leur forte valeur nutritionnelle. De nombreux travaux ont montré qu'un régime à base de protéines de soja était hypocholestérolémiant par rapport à un régime à base de caséine, chez l'animal comme chez l'homme (Nagata et al, 1982; Terpstra et al, 1983; Forsythe et al, 1986; Kritchevsky et al, 1987). De ce fait, les protéines de soja intéresseraient les pays occidentaux pour leur capacité à diminuer le risque de maladies cardiovasculaires.

De leur côté, les alginates et carraghénates alimentaires ne sont inertes ni sur le plan chimique, ni sur le plan physiologique (Adrian et Assoumani, 1979). Dans le tube digestif, ils réagissent avec les protéines en les rendant partiellement indisponibles et peuvent de ce fait causer des retards de croissance. C'est le cas, chez le rat, lors de l'ingestion de régimes contenant $5 \%$ d'alginate de sodium ou 10 à $25 \%$ de carraghénate de sodium (Assoumani et Adrian, 1976; Nilson and Wagner, 1959). $\mathrm{De}$ plus, les carraghénates, comme les fibres solubles telles que la pectine, la gomme guar et la gomme xanthane, présentent un effet hypolipémiant et antiathérogénique (Ershoff and Wells, 1962; Kritchevsky and Story, 1986).

L'association protéines de sojahydrocolloïdes alimentaires serait donc susceptible de provoquer des perturbations de l'absorption intestinale, dont les conséquences seraient un développement ralenti des animaux et une modification du métabolisme lipidique.

Des résultats précédents ont montré que l'ingestion de mélanges chauffés de farine de soja associée à $3 \%$ d'alginate ou de $0,5,1,2$ ou $3 \%$ de carraghénate de sodium entraînait un coefficient d'efficacité protéique (CEP) inférieur à celui obtenu après ingestion de caséine, confirmant ainsi le rôle précipitant des carraghénates sur les protéines (Mouecoucou et al, 1990). Nous avons poursuivi cette expérience en étudiant l'évolution pondérale et le métabolisme lipidique des mêmes animaux, lorsqu'ils sont réalimentés avec un régime standard pendant 16 semaines, puis avec un régime à $20 \%$ de protéines 
fournies par la farine de soja associée à l'alginate ou au carraghénate de sodium pendant 6 semaines.

\section{MATÉRIELS ET MÉTHODES}

\section{Étude expérimentale}

Des rats mâles de souche Wistar (IFFA CREDO, Saint-Germain sur l'Arbresle, France) âgés de $21 \mathrm{j}$, et pesant entre 45 et $50 \mathrm{~g}$ au début de l'expérimentation ont été nourris dans un premier temps pendant 4 sem avec des régimes à $10 \%$ de protéines de caséine (UAR, Villemoisson/Orge, France) ou de soja associées ou non à l'alginate ou au carraghénate de sodium à des taux de $0,5,1,2$ ou $3 \%$ (soit 0,10 , $0,21,0,42$ ou $0,63 \%$ d'alginate ou de carraghénate de sodium dans les régimes). Après cette période, tous les lots de rats ont reçu pendant 16 sem un régime standard comportant $17 \%$ de protéines. A l'issue de cette deuxième période expérimentale, les rats des lots constitués lors de la première période ont été réalimentés pendant $6 \mathrm{sem}$ avec les mêmes régimes que ceux de la première période à base de caséine ou de farine de soja associée ou non aux hydrocolloïdes alimentaires, mais comprenant cette fois $20 \%$ de protéines.

La première période avait pour but l'étude de l'influence des interactions entre les protéines de soja et les épaississants sur le coefficient d'efficacité protéique et les résultats ont été publiés (Mouecoucou et al, 1990). Lors de la deuxième période expérimentale, nous avons observé les effets de la renutrition sur l'évolution pondérale des rats des différents lots. La troisième période expérimentale avait pour but d'étudier les effets de l'alginate et du carraghénate de sodium sur les paramètres lipidiques de ces rats renutris. Pour ce faire, il fallait utiliser des régimes dont les taux de protéines étaient comparables à ceux utilisés habituellement dans des travaux analogues.

Durant toute l'étude, l'évolution pondérale des animaux a été mesurée. A la fin de l'expérimentation, les rats ont été sacrifiés à jeun par décapitation après anesthésie à l'éther. Le sang a été recueilli sur anticoagulant $\left(\mathrm{Na}_{2} \mathrm{EDTA}\right.$ Iniprol).

\section{Nature des régimes}

Les régimes utilisés ont varié selon les périodes expérimentales. Leurs compositions sont reproduites dans les tableaux I et II. Au cours des première et troisième périodes, un régime à base de caséine et 9 régimes à base de farine déshuilée de soja (Ets Louis François, St Maur, France) ont été préparés. Cette farine a été additionnée de $0,5,1,2$ ou $3 \%$ d'alginate ou de carraghénate de sodium (Ets Louis François, St Maur, France) et chauffée. Selon les périodes, la quantité de farine nécessaire pour avoir $10 \mathrm{ou}$ $20 \%$ de protéines a été pesée, le contenu en lipides de chaque régime a été ajusté à 9 ou $5 \%$ avec de l'huile d'arachide (Lesieur) en tenant compte du pourcentage de lipides contenu dans les farines. La composition physico-chimique de cette farine et le mode de préparation des régimes ont été décrits précédemment (Mouecoucou et al, 1990). Au cours de la deuxième période, tous les rats ont reçu le même régime standard (UAR, Villemoisson/Orge, France).

\section{Dosages}

Le sang recueilli sur anticoagulant a été centrifugé à $2800 \mathrm{~g}$ pendant $20 \mathrm{~min}$ pour récupérer le plasma. Les lipoprotéines plasmatiques ont été préparées par ultracentrifugation selon la méthode de Havel et al (1955).

Les dosages de la cholestérolémie (CT) et de la triglycéridémie (TG) ont été effectués sur le plasma et sur les différentes fractions par des méthodes enzymatiques à l'aide de trousses commercialisées par Boehringer Mannheim.

\section{Tests statistiques}

Les résultats obtenus sur les différents lots de rats ont été comparés par analyse de variance à une voie, et par un test de Fisher au seuil de $5 \%$ à l'aide d'un programme Statview sur un microordinateur Macintosh. 
Tableau I. Composition des régimes de base pour chaque période.

\begin{tabular}{|c|c|c|c|}
\hline Composition & $\begin{array}{l}\text { 1re période } \\
\text { (\%) }\end{array}$ & $\begin{array}{c}2^{\theta} \text { période } \\
\left.\text { (régime standard UAR n }{ }^{\circ} 4\right) \\
(\%)\end{array}$ & $\begin{array}{c}3^{\theta} \text { période } \\
\text { (\%) }\end{array}$ \\
\hline Protéines & 10 & $17^{\star \star \star \star}$ & 20 \\
\hline Lipides (huile d'arachide) & 9 & 3 & 5 \\
\hline Cellulose & 5 & 4 & 5 \\
\hline Mélange minéral ${ }^{\star}$ & 5 & 4,30 & 5 \\
\hline Mélange vitaminique** & 2 & - & 2 \\
\hline Eau & - & 12 & - \\
\hline Amidon & 69 & 58,70 & 63 \\
\hline
\end{tabular}

- Composition du mélange salin : CaHPO $4: 430 \mathrm{~g}, \mathrm{KCl}: 100 \mathrm{~g}, \mathrm{NaCl}: 100 \mathrm{~g}, \mathrm{MgO}: 10,5 \mathrm{~g}, \mathrm{MgSO}_{4}: 50 \mathrm{~g}_{1} \mathrm{Fe}_{2} \mathrm{O}_{3}: 3$ g. $\mathrm{Fe} \mathrm{SO}_{4} 7 \mathrm{H}_{2} \mathrm{O}: 5 \mathrm{~g}$; oligoéléments : $10 \mathrm{~g}$. QSP : $1000 \mathrm{~g}$. "* Composition du mélange vitaminique : tocophérol : 11 $\mathrm{mg}$, Niacine : $10 \mathrm{mg}$, pantothénate de calcium : $6 \mathrm{mg}$, vitamine A : $2000 \mathrm{Ul}$, chlorure de choline : $166 \mathrm{mg}$, riboflavine : $2 \mathrm{mg}$, vitamine $B_{12}: 0,03 \mathrm{mg}$, menadione : $5 \mathrm{mg}$, inositol : $11 \mathrm{mg}$, chlorhydrate de pyrodoxine : $2 \mathrm{mg}$, acide folique : $0,2 \mathrm{mg}$, acide ascorbique : $100 \mathrm{mg}$, acide $\mathrm{p}$-aminobenzoïque : 11 , thiamine : $2 \mathrm{mg}$, vitamine $\mathrm{D}: 200 \mathrm{UI}$, biotine : $0,04 \mathrm{mg}$. *** Les protéines du régime UAR $n^{\circ} 4$ utilisé lors de la seconde période expérimentale sont à base de céréales (88\%) de tourteaux de protéines végétales $(7 \%)$, de protéines de poisson $(2 \%)$, et d'un mélange minéral vitaminé $(3 \%)$.

Tableau II. Nature des protéines composant les régimes lors des première et troisième périodes.

\section{Régimes}

$\begin{aligned} 1 & \text { Caséine } \\ 2 & \text { Farine de soja chauffée* } \\ 3 & \text { Farine de soja }+0,5 \% \text { d'alginate de sodium } \\ 4 & \text { Farine de soja }+1 \% \text { d'alginate de sodium } \\ 5 & \text { Farine de soja }+2 \% \text { d'alginate de sodium } \\ 6 & \text { Farine de soja }+3 \% \text { d'alginate de sodium } \\ 7 & \text { Farine de soja }+0,5 \% \text { de carraghénate de sodium } \\ 8 & \text { Farine de soja }+1 \% \text { de carraghénate de sodium } \\ 9 & \text { Farine de soja }+2 \% \text { de carraghénate de sodium } \\ 10 & \text { Farine de soja }+3 \% \text { de carraghénate de sodium }\end{aligned}$

* Composition de la farine de soja (\%) : Protéine 48,17; Lipides 3,93; glucides éthanolosolubles 10,63, humidité 7,39; cendres 6,16 . " La farine de soja a été associée aux hydrocolloïdes, ce mélange a été chauffé. 


\section{RÉSULTATS}

\section{Évolution pondérale des rats} nourris avec des régimes à base d'alginate (fig 1)

La figure 1 montre l'évolution pondérale des rats nourris avec des mélanges chauffés de farine de soja plus de l'alginate de sodium à différents taux, comparés à ceux nourris avec de la caséine ou avec de la farine de soja chauffée. II n'y a aucune dif- férence significative entre les lots dans aucune des trois périodes expérimentales.

\section{Évolution pondérale des rats nourris avec des régimes à base de carraghénate (fig 2)}

La figure 2 illustre l'évolution pondérale des rats nourris avec de la caséine ou de la farine de soja chauffée, ou de la farine de soja mélangée au carraghénate de sodium à différents taux et chauffée. Quand

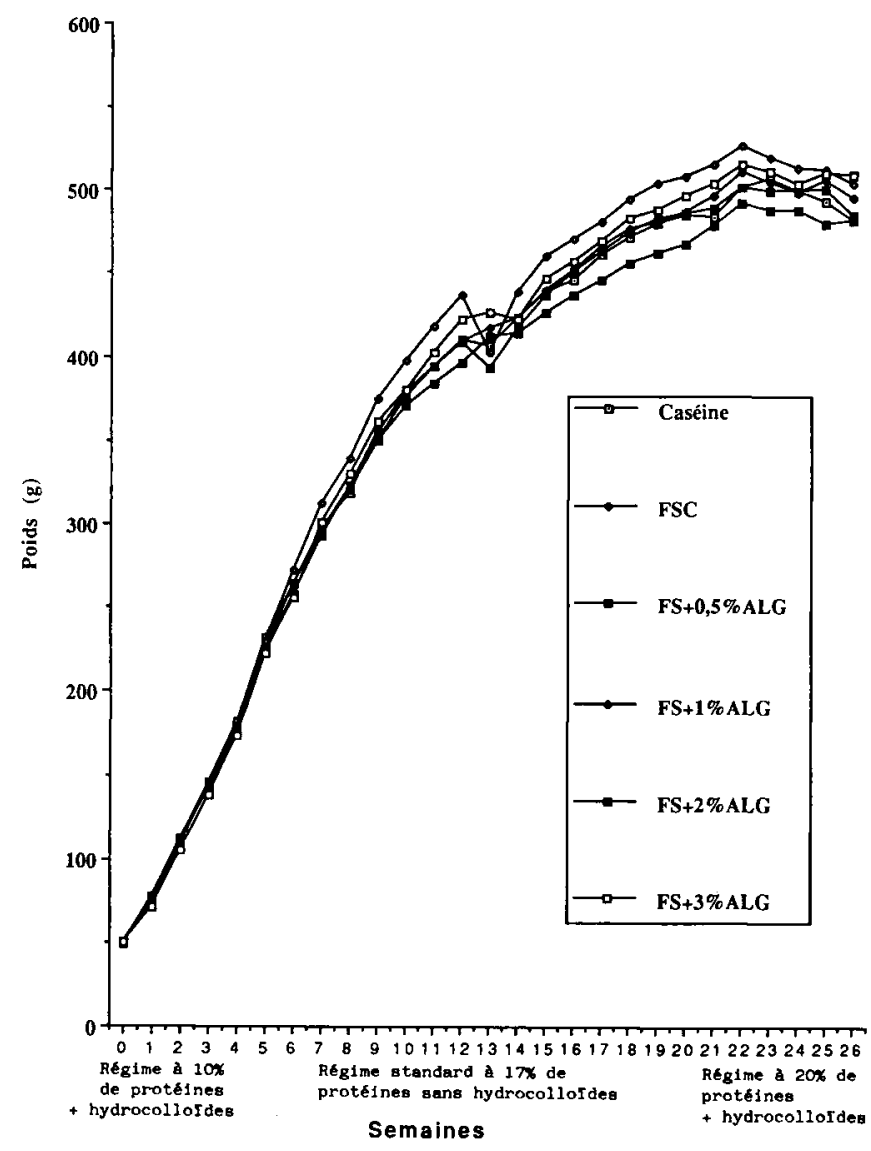

Fig 1. Effet des doses croissantes d'alginates sur l'évolution pondérale des rats. 


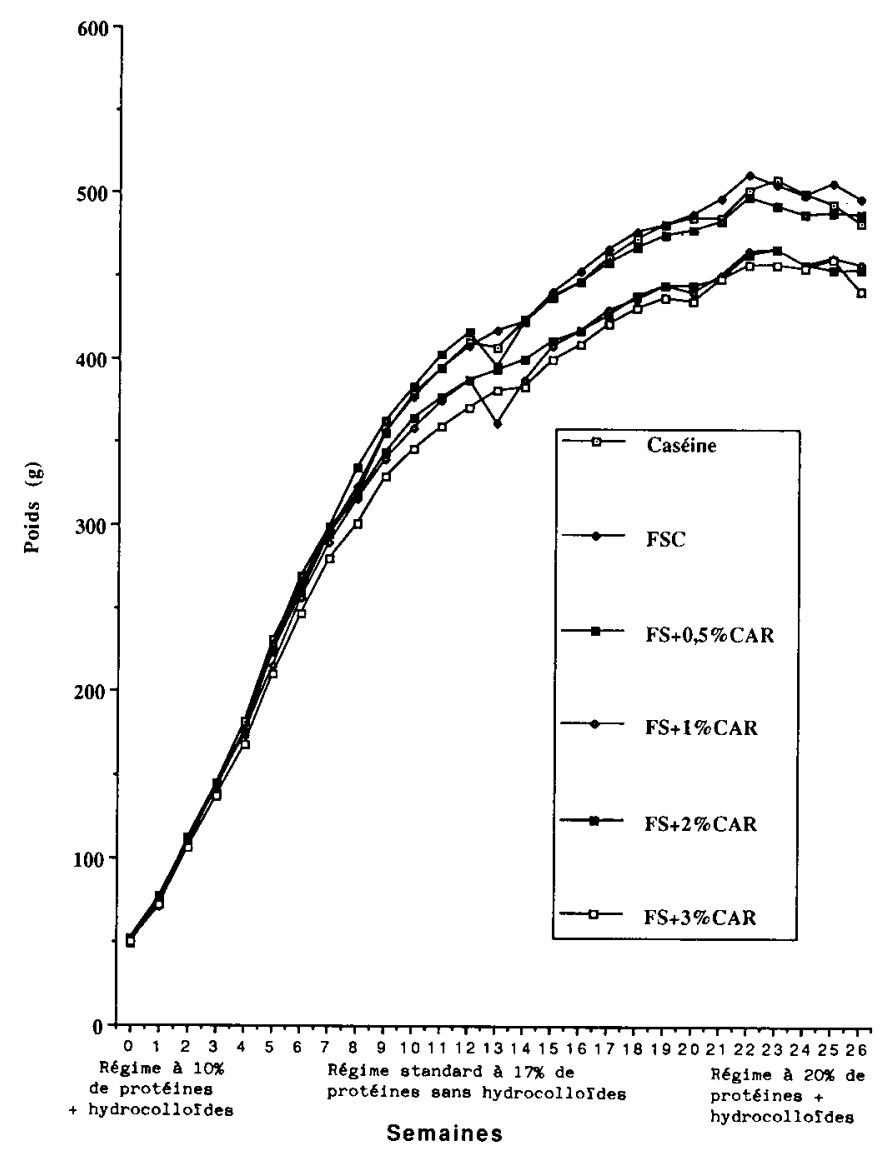

Fig 2. Effet des doses croissantes de carraghénates sur l'évolution pondérale des rats.

les rats sont réalimentés avec le régime standard, seuls les rats nourris auparavant avec de la farine de soja contenant $0,5 \%$ de carraghénate présentent la même évolution pondérale que les groupes nourris avec de la caséine ou de la farine de soja chauffée. En revanche, les rats nourris au préalable avec de la farine de soja additionnée de 1,2 ou $3 \%$ de carraghénates montrent une croissance inférieure à celle des 3 groupes précédents. Pour les lots ayant reçu $3 \%$ de carraghénate, la différence de poids est significative $(P<0,05)$ dès la première semaine de régime standard (5e semaine de l'expérimentation). Pour tous les autres groupes, elle apparaît à partir de la $9^{e}$ semaine de régime standard et est maintenue jusqu'à la fin de l'expérimentation. Dans tous les cas, le passage à un régime à $20 \%$ de protéines n'entraîne pas de modification de cette courbe de poids. 


\section{Effets de régimes à base d'alginate de sodium sur la triglycéridémie et sur la cholestérolémie des rats (tableaux III et IV)}

II n'y a pas de variation significative des triglycérides plasmatiques dans les groupes de rats nourris avec de la caséine, de la farine de soja associée ou non à $0,5,2$ ou $3 \%$ d'alginate de sodium. Un effet hypo- cholestérolémiant est observé avec $1 \%$ d'alginate dans la farine de soja (tableau III) par comparaison avec tous les autres régimes. Le taux de triglycérides dans les VLDL est le reflet des taux des triglycérides plasmatiques, alors que les triglycérides des fractions LDL et HDL ne varient pas.

Un effet hypercholestérolémiant de la farine de soja chauffée et supplémentée

Tableau III. Effets de l'alginate de sodium sur la triglycéridémie des rats (moyenne \pm écart type).

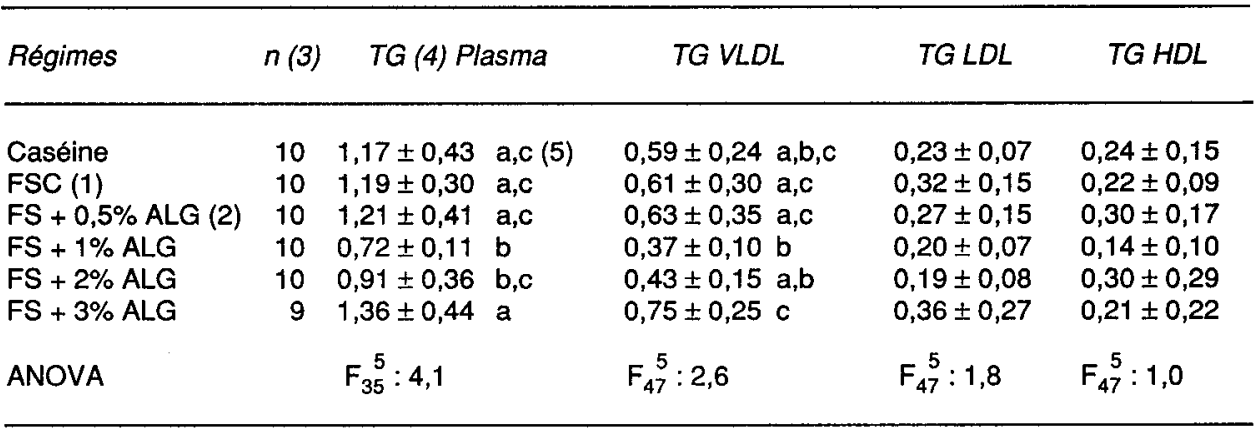

(1) FSC : farine de soja chauffée, (2) ALG : alginate de sodium, (3) $n$ : nombre d'animaux par groupe, (4) TG : triglycérides, (5), Dans chaque colonne, des couples de résultats sont significativement différents au seuil de $5 \%$ quand aucune lettre n'est commune. Une colonne ne comporte pas de lettre quand l'analyse de variance n'a pas décelé de significativité globale.

Tableau IV. Effets de l'alginate de sodium sur la cholestérolémie des rats (moyenne \pm écart type).

\begin{tabular}{|c|c|c|c|c|c|}
\hline Régimes & $n$ & CT (6) Plasma & $C T V L D L$ & $C T L D L$ & CTHDL \\
\hline $\begin{array}{l}\text { Caséine } \\
\text { FSC } \\
\text { FS + 0,5\% ALG } \\
\text { FS + } 1 \% \text { ALG } \\
\text { FS + } 2 \% \text { ALG } \\
\text { FS + } 3 \% \text { ALG }\end{array}$ & $\begin{array}{r}7 \\
9 \\
10 \\
9 \\
9 \\
9\end{array}$ & $\begin{array}{l}0,48 \pm 0,16 \text { a } \\
0,63 \pm 0,13 \text { b,c } \\
0,67 \pm 0,16 \quad b \\
0,64 \pm 0,13 \text { b,d } \\
0,52 \pm 0,17 \text { a,c,d } \\
0,68 \pm 0,14 \quad b\end{array}$ & $\begin{array}{l}0,07 \pm 0,05 \\
0,07 \pm 0,03 \\
0,10 \pm 0,05 \\
0,07 \pm 0,03 \\
0,07 \pm 0,05 \\
0,10 \pm 0,04\end{array}$ & $\begin{array}{l}0,12 \pm 0,06 \\
0,16 \pm 0,04 \\
0,14 \pm 0,04 \\
0,18 \pm 0,07 \\
0,13 \pm 0,06 \\
0,18 \pm 0,08\end{array}$ & $\begin{array}{ll}0,26 \pm 0,06 & a \\
0,32 \pm 0,07 & a, c \\
0,38 \pm 0,09 & b, c \\
0,44 \pm 0,10 & c \\
0,35 \pm 0,10 & b \\
0,32 \pm 0,05 & a, b\end{array}$ \\
\hline ANOVA & & $\mathrm{F}_{47}^{5}: 2,7$ & $F_{47}^{5}: 1,3$ & $F_{47}^{5}: 1,7$ & $F_{47}^{5}: 4,8$ \\
\hline
\end{tabular}

(6) CT : cholestérol 
ou non par des alginates est observé par rapport à la caséine (tableau IV) sauf avec le mélange chauffé de farine de soja associée à $2 \%$ d'alginate. Le cholestérol ne varie ni dans les VLDL, ni dans les LDL des différents groupes de rats. Le cholestérol des HDL varie dans le même sens que celui du plasma et augmente de façon significative dans les groupes nourris avec de la farine de soja supplémentée en alginates par rapport à la caséine sauf pour la farine de soja chauffée et pour la farine de soja mélangée avec $3 \%$ d'alginate et chauffée.

\section{Effets des régimes à base de carraghénate de sodium sur la triglycéridémie et la cholestérolé- mie des rats (tableaux $V$ et $V I$ )}

II n'y a pas de différence significative des triglycérides plasmatiques entre les groupes alimentés avec de la caséine ou avec de la farine de soja contenant ou non $0,5 \%$ de carraghénate et chauffée (tableau $V)$. En revanche, on observe un effet hy- potriglycéridémiant significatif $(P<0,05\rangle$ des carraghénates quand ils sont présents dans la farine de soja aux taux de 1,2 ou $3 \%$ par rapport aux groupes précédents. Cet effet hypotriglycéridémiant est retrouvé dans toutes les fractions lipoprotéiques et semble en relation avec la dose de carraghénate utilisée. II paraît cependant plus marqué pour les VLDL et les HDL.

L'analyse de variance montre qu'il n'existe pas d'effet global dû au régime sur la cholestérolémie (tableau VI). L'augmentation de la cholestérolémie observée dans les groupes farine de soja chauffée et farine de soja contenant $0,5 \%$ de carraghénate et chauffée est due à la farine de soja. Cet effet de la farine de soja est retrouvé au cours de l'étude du cholestérol des HDL.

\section{DISCUSSION}

Les animaux nourris après leur sevrage avec de la farine de soja ont présenté, par la suite, une évolution pondérale différente selon que leur régime initial contenait de

Tableau V. Effets du carraghénate de sodium sur la triglycéridémie des rats (moyenne \pm écart type).

\begin{tabular}{|c|c|c|c|c|c|}
\hline Régimes & $n(3)$ & TG (4) Plasma & $T G V L D L$ & $T G L D L$ & $T G H D L$ \\
\hline $\begin{array}{l}\text { Caséine } \\
\text { FSC (1) } \\
\text { FS + } 0,5 \% \text { CAR (2) } \\
\text { FS }+1 \% \text { CAR } \\
\text { FS }+2 \% \text { CAR } \\
\text { FS }+3 \% \text { CAR }\end{array}$ & $\begin{array}{r}10 \\
10 \\
9 \\
8 \\
10 \\
10\end{array}$ & $\begin{array}{l}1,17 \pm 0,43 \text { a }(5) \\
1,19 \pm 0,30 \text { a } \\
1,16 \pm 0,32 \text { a } \\
0,80 \pm 0,31 \text { b } \\
0,60 \pm 0,16 b \\
0,63 \pm 0,25 b\end{array}$ & $\begin{array}{l}0,59 \pm 0,24 \quad a \\
0,61 \pm 0,30 \quad a, b \\
0,81 \pm 0,17 \quad b \\
0,42 \pm 0,16 a, c \\
0,34 \pm 0,12 \quad c \\
0,36 \pm 0,21 \quad c\end{array}$ & $\begin{array}{l}0,23 \pm 0,07 \quad a, b \\
0,32 \pm 0,15 \quad a \\
0,21 \pm 0,07 \quad a, b \\
0,23 \pm 0,20 a, b \\
0,15 \pm 0,08 \quad b \\
0,14 \pm 0,08 \quad b\end{array}$ & $\begin{array}{l}0,24 \pm 0,15 a \\
0,22 \pm 0,09 a, b \\
0,23 \pm 0,13 a, b \\
0,11 \pm 0,07 c \\
0,09 \pm 0,07 c \\
0,13 \pm 0,12 b, c\end{array}$ \\
\hline ANOVA & & $F_{51}^{5}=8,2$ & $F_{48}^{5}=5,6$ & $F_{48}^{5}=2,9$ & $F_{48}^{5}=3,7$ \\
\hline
\end{tabular}

(1) FSC : farine de soja chauffée, (2) CAR : carraghénate de sodium, (3) $n$ : nombre d'animaux par groupe, (4) TG : triglycérides, (5) Dans chaque colonne, des couples de résultats sont significativement différents au seuil de $5 \%$ quand aucune lettre n'est commune. Une colonne ne comporte pas de lettre quand l'analyse de variance n'a pas décelé de significativité globale. 
Tableau VI. Effets du carraghénate de sodium sur la cholestérolémie des rats (moyenne \pm écart type).

\begin{tabular}{lrlllll}
\hline Régimes & $n$ & CT (6) Plasma & CT VLDL & CTLDL & CT HDL \\
\hline Caséine & 7 & $0,48 \pm 0,16$ & $0,07 \pm 0,05$ & $0,12 \pm 0,06$ & $0,26 \pm 0,06$ & a \\
FSC & 9 & $0,63 \pm 0,13$ & $0,07 \pm 0,03$ & $0,16 \pm 0,04$ & $0,32 \pm 0,07$ & a \\
FS + 0,5\% CAR & 8 & $0,63 \pm 0,16$ & $0,10 \pm 0,03$ & $0,18 \pm 0,07$ & $0,37 \pm 0,09$ & b \\
FS + 1\% CAR & 9 & $0,59 \pm 0,15$ & $0,07 \pm 0,04$ & $0,14 \pm 0,05$ & $0,35 \pm 0,04$ & b \\
FS+ 2\% CAR & 10 & $0,60 \pm 0,15$ & $0,09 \pm 0,04$ & $0,15 \pm 0,07$ & $0,33 \pm 0,08$ & b \\
FS + 3\% CAR & 10 & $0,58 \pm 0,11$ & $0,09 \pm 0,05$ & $0,12 \pm 0,03$ & $0,38 \pm 0,08$ & b \\
ANOVA & & $F_{47}^{5}=1,2$ & $F_{47}^{5}=1,0$ & $F_{47}^{5}=1,6$ & $F_{47}^{5}=3,0$ & \\
\hline
\end{tabular}

(6) CT : cholesterol.

l'alginate ou du carraghénate de sodium. Alors que les lots de rats ayant reçu des alginates montraient une évolution pondérale normale et identique à celle des rats nourris avec de la caséine lorsqu'on les alimentait avec un régime standard, un retard de croissance était remarqué avec les animaux dont la farine de soja composant leur régime était supplémentée de 1,2 ou $3 \%$ de carraghénate. La faible croissance de ces animaux serait due à l'association du carraghénate et de la farine de soja, le tout étant chauffé. Plusieurs travaux ont montré un effet antiprotéolytique des carraghénates, particulièrement un effet antipepsique (Anderson, 1961; Accary et al, 1964; Martin et al, 1965), et une activité antitrypsique (Gatfield et Stute, 1972). De plus, il a été prouvé que, par rapport aux alginates et à la gomme guar, les carraghénates se liaient plus fortement aux protéines et avaient une viscosité plus élevée (Tsai, 1989). À pH inférieur au point isoélectrique des protéines et en présence de cations tels que le potassium et le calcium, les carraghénates insolubilisaient les protéines, ce qui n'était pas le cas des alginates (Adrian et Assoumani, 1979). Tous ces éléments suggèrent qu'en présence des carraghénates, il se produirait une diminution de la digestibilité des protéines. A cela s'ajoute le fait que les protéines de soja sont légèrement déficientes en méthionine. Comme le taux de protéines est faible $(10 \%)$ dans l'étude du CEP, et comme le taux de méthionine des protéines de soja $(1,5 \mathrm{~g} / 16 \mathrm{~g}$ d'azote) est faible comparé à celui de la caséine $(2,5 \mathrm{~g} /$ $16 \mathrm{~g}$ d'azote), la quantité de méthionine est juste suffisante dans le cas de la farine de soja chauffée (CEP : 2,25; Mouecoucou et al, 1990). Si une partie des protéines est insolubilisée ou rendue indisponible par les carraghénates, par un mécanisme qui reste à élucider, alors que la quantité de méthionine disponible pour l'anabolisme pourrait être insuffisante, ceci pourrait engendrer une malnutrition protéique par déficience en cet acide aminé essentiel.

II est bien connu que les animaux ayant subi une malnutrition protéique précoce ou nés de mères carencées pendant la gestation ont un retard de croissance qui n'est plus jamais rattrapé malgré le retour à une alimentation équilibrée. Cet effet est d'autant plus marqué que le temps de privation est long (Dickerson et al, 1972; Nolen, 1972; Harris, 1980; Okasaki et al, 1981; 
Tulp et Norton, 1981; Beck et al, 1983; Warren et Bedi, 1985). Le fait de nourrir les rats dès leur sevrage avec un régime comportant peu de méthionine et dont la disponibilité protéique est réduite par la présence de carraghénates pourrait expliquer en partie nos résultats.

Nilson et Wagner (1959) ont montré que les carraghénates présents à des taux inférieurs ou égaux à $10 \%$ dans les régimes à base de caséine non chauffée ne provoquaient pas de retard de croissance des animaux. Or, notre expérience montre que la présence de carraghénate à partir de $0,21 \%$ dans le régime des animaux entraîne un ralentissement de la croissance de ces derniers. II est possible que le chauffage du mélange farine de sojacarraghénate augmente aussi l'indisponibilité des protéines.

Nos résultats montrent un effet hypotriglycéridémiant obtenu avec l'addition de $0,21 \%$ ou plus de carraghénate de sodium dans le régime. Cette diminution semble proportionnelle à la dose ajoutée. Les taux utilisés ici sont très faibles par rapport à ceux généralement connus comme présentant un effet hypolipémiant. Nous n'avons pas retrouvé l'activité anticholestérolémique et antiathérogénique des carraghénates observée par Fahrenbach et al (1966) chez le poulet avec des doses de 1 à $3 \%$ de carraghénates dans les régimes, et par Ershoff et Wells (1962) chez les rats ingérant un régime supplémenté avec $1 \%$ de cholestérol et contenant $10 \%$ de carraghénates. Cet effet hypocholestérolémiant a été également observé avec d'autres hydrocolloïdes tels que la pectine, la gomme guar, la gomme xanthane, etc. (Keys et al, 1961; Ershoff et Wells, 1962; Kiriyama et al, 1969; Creger et al, 1970). Ces substances agiraient en inhibant la réabsorption intestinale des sels biliaires, réduisant ainsi les possibilités d'absorption intesti- nale de tous les constituants lipophiles (Fisher et al, 1964) et notamment du cholestérol dont le taux plasmatique se trouverait ainsi diminué.

L'effet hypotriglycéridémiant de $1 \%$ d'alginate de sodium additionné à de la farine de soja alors qu'aucun effet significatif n'a été noté pour les autres doses employées est difficile à expliquer et mérite d'être vérifié. L'augmentation de la cholestérolémie sous l'effet des régimes à base de farine de soja chauffée par rapport à la caséine n'est pas un effet propre des alginates, mais plutôt un effet de la nature des protéines, le même type de résultats étant obtenu avec les carraghénates.

L'effet hypocholestérolémiant généralement observé avec les protéines végétales, et particulièrement avec les protéines de soja par rapport à la caséine, n'est pas observé ici. Au contraire, un effet hypercholestérolémiant relatif de la farine de soja par rapport à la caséine a été remarqué. Les dosages ayant mis en évidence l'effet hypocholestérolémiant du soja par rapport à la caséine chez le rat Wistar ont été effectués, pour la plupart d'entre eux, sur des prélèvements réalisés sur des animaux nourris ou ayant subi un jeûne de courte durée. Des travaux réalisés sur le porc ont montré l'influence de l'état nutritionnel sur les concentrations sanguines de cholestérol et de triglycérides (Beynen et al, 1990) en fonction du type de protéine ingérée. Dans le cas présent, nos rats sont à jeun depuis la veille à $17 \mathrm{~h}$. Les conditions expérimentales de l'étude de l'effet de la nature des protéines sont très variables (souche des animaux, durée des expérimentations, état nutritionnel des animaux). Elles pourraient fournir une explication à l'absence d'hypocholestérolémie observée après ingestion de soja dans ce travail. 


\section{CONCLUSION}

Les effets observés sur la croissance et la triglycéridémie pourraient être dus aux propriétés physico-chimiques du produit obtenu après chauffage du mélange comprenant des protéines de soja et du carraghénate de sodium.

\section{RÉFÉRENCES}

Accary JP, Dubrasquet M, Agneray J, Bonfils S (1964) Étude électrophorétique du complexe polysaccharides sulfates-protéines. $C R$ SoC Biol 158, 1844-1847

Adrian J, Assoumani M (1979) Les propriétés chimiques et physiologiques des additifs épaississants et gélifiants (agents de texture). Rev Fr Diet 23, 3-25

Assoumani M, Adrian J (1976) Les alginanes en alimentation. Étude chez le rat. AlimVie 64, 45-55

Anderson W (1961) The antipeptic activity of sulfated polysaccharides. J Pharm Pharmacol 13, 139-147

Beck B, Dollet JM, Max JP, Debry G (1983) Relations among weight deficit, food intake and early deprivation in long-term protein malnourished rats. Nutr Res 3, 743-748

Beynen AC, West CE, Spaaij CJK, Huisman J, Leeuwen $P$, Van Schutte J Ben, Hackeng WHL (1990) Cholesterol metabolism, digestion rates and postprandial changes in serum of swine fed purified diets containing either casein or soybean protein. J Nutr 120, 422430

Creger CR, Guzman VA de, Couch JR (1970) Effect of guar gum on cholesterol levels of chickens. Nutr Rep Int 2, 243-247

Dickerson JWT, Hugues PCR, Mc Anulty PA (1972) The growth and development of rats given a low protein diet. Br J Nutr 27, 527

Ershoff BH, Wells AF (1962) Effects of methoxyl content on anticholesterol activity of peptic substances in the rats. Expl Med Surg 20, 272-276
Fahrenbach MJ, Riccardi BA, Grant WC (1966) Hypocholesterolemic activity of mucilaginous polysaccharides in white Leghorn cockerels. Proc Soc Exp Biol Med 123, 321-326

Fisher H, Griminger P, Weiss HS (1964) Avian atherosclerosis: retardation by pectin. Science 146, 1063-1064

Forsythe WA, Green MS, Anderson JJB (1986) Dietary protein effects on cholesterol and lipoprotein concentrations: a review. J Am Coll Nutr 5, 533-549

Gatfield IL, Stute R (1972) Enzymatic reaction in the presence of polymers. The competitive inhibition of trypsin by lambda-carrageenan. Febs Lett 28, 29-31

Harris $P(1980)$ Changes in adipose tissue of the rat due to early undernutrition followed by rehabilitation. 1. Body composition and adipose tissue cellularity. Br J Nutr 43, 15-26

Havel RJ, Eder H, Bradgon JH (1955) The distribution and chemical composition of ultracentrifugally separated lipoproteins in human serum. J Clin Nutr 34, 1345-1353

Keys A, Grange F, Anderson JT (1961) Fiber and pectin in the diet and serum cholesterol concentration in man. Proc Soc Exp Biol Med 106, 555-558

Kiriyama S, Ohazaki Y, Yoshida A (1969) Hypocholesterolemic effect of polysaccharides and polysaccharide-rich foodstuff in cholesterol fed rats. $J$ Nutr $97,382-388$

Kritchesvky D, Story JA (1986) Influence of dietary fiber on cholesterol metabolism in experimental animals. In: Handbook of Dietary Fiber in Human Nutrition (Spiller GA, ed) Boca Raton, FI : CRC, 129-142

Kritchevsky D, Tepper SA, Klurfeld DM (1987) Dietary protein and atherosclerosis. J Am Oil Chem Soc 64, 1167

Martin F, Bérard A, Vagne M, Lambert R (1965) Étude électrophorétique de l'action des différents polysaccharides sulfatés sur la protéolyse peptique. CR Soc Biol 159, 1582-1585

Mouecoucou J, Villaume C, Bau HM, Nicolas JP, Mejean L (1990) Effets des alginates et des carraghénates de sodium associés aux protéines de soja sur le coefficient d'efficacité protéique (CEP). Rep Nutr Dóv 30, 541-547

Nagata $Y$, Ishiwaki N, Sugano M (1982) Studies on the mechanism of antihypercholesterolemic action of soy protein and soy protein-type 
amino acid mixtures in relation to the casein counterparts in rats. $J$ Nutr 112, 1614-1625

Nilson and Wagner (1959) Feeding test with carrageenin. Foods Res 24, 235-239

Nolen GA (1972) Effect of various restricted dietary regimes on the growth, health and longevity of albino rats. $J$ Nutr 102, 1477-1494

Okasaki S, Matsueda S, Ohnaka M, Niiyama Y (1981) Effect of various periods of protein restriction immediately after weaning on subsequent catch-up growth in rats. Nutr Rep int 23, 471-484

Terpstra AHM, Hermus RJJ, West CE (1983) The role of dietary protein in cholesterol metabolism in rabbits and rats. In: Animal and Vegetable Proteins in Lipid Metabolism and
Atherosclerosis. (MJ Gibney, D Kritchevsky, eds) Alan R Liss, Inc, New York, 19-49

Tsai S, Yamada K, Murakami $H$, Omuka $H$ (1989) Effect of polysaccharides on textural characteristic of soy protein isolate emulsion. Nippon Shokuhin Kogyo Gakkaishi 36, 765768

Tulp OL, Horton ES (1981) Effect of prolonged experimental protein malnutrition and on refeeding on growth, adipose tissue development and body composition in rats. $J$ Nutr 111, 1145-1156

Warren MA, Bedi KS (1985) The effect of lenghty period of undernutrition on food intake and on body and organs growth during rehabilitation. J Anat 141, 65-75 